\title{
Articles
}

CARMEN CORONA MARZOL ${ }^{1}$

\section{Conocimientos forales y saberes de viudas. Felipa Clavero Sessé ante la ley por el condado de Aranda durante el reinado de Carlos $\mathrm{II}^{2}$}

\author{
Foral knowledges and widow's wisdom. Felipa Clavero \\ Sessé before the law for the Aranda County during the \\ reign of Carlos II
}

\section{RESUMEN}

Este artículo tiene como escenario nobiliario el condado de Aranda en la segunda mitad del seiscientos. Profundizando en el marco que aportaban las condiciones de la viudez, y en las contradicciones y desafíos de los derechos legítimos de las viudas. Nuestro estudio se centra en una de las condesas más representativas de la Casa y Estado de Aranda, doña Felipa Clavero y Sessé, desprotegida de sus derechos legítimos a favor de otro aspirante al condado y a la herencia mencionada. En un proceloso mar de tribunales se representan los intereses familiares, así como los del entorno parental y clientelar a los cuales se unen los que provienen de agendas políticas y económicas diversas.

Palabras clave: Derechos de viudas, conocimientos forales, condado de Aranda, Felipa Clavero y Sessé, Reinado de Carlos II.

\begin{abstract}
This article has as a noble scenery the Aranda County during the second half of the $17^{\text {th }}$ century, digging into the framework that granted the widowhood, and into the contradictions and challenges of the legitimate rights of the widows. Our study focuses in one of the more representative duchesses of the Aranda House and State, doña Felipa Clavero y Sessé, whose own and legitimate rights were taken from her, in favour of another suitor to the County and to the mentioned inheritance. The family, parental and client interests join the different economic and political agendas in a stormy sea of courts.

Keywords: Widows rights, foral knowledges, Aranda County, Felipa Clavero y Sessé, Carlos II reign.
\end{abstract}

\section{SUMARIO}

1. La condición de viudez en el entramado político aragonés. 2. La V condesa de Aranda. 3. Felipa Clavero y Sessé: la mujer. 4. Los condicionantes forales de los litigios judiciales en Aragón. 5. El problema de toda una vida: los derechos de viudedad de Felipa Clavero y Sessé. 6. Conclusiones.

1 Catedrática acreditada de Historia Moderna de la Universitat Jaume I; corona@uji.es

2 Este trabajo se ha realizado dentro del proyecto de investigación, financiado por la Universitat Jaume I: De pequeños hidalgos a nobles titulados. Riquezas, poder y redes clientelares de la nobleza mediterránea (P1. 1A2014-13). 


\section{La condición de viudez en el entramado político aragonés}

Los derechos de viudedad de las mujeres de la nobleza es un tema conocido, que cuenta con análisis generales entre los estudios de género. Partimos del conocimiento común y de las consideraciones generales ya conocidas sobre las viudas en el Antiguo Régimen.

Este estudio se centra en el caso concreto de las vicisitudes de la condesa Felipa Clavero y Sessé y sus interminables pleitos en el mar de los tribunales por la posesión de sus derechos de viudedad. En ellos se dan cita diversos pretendientes interesados en el legado de las propiedades heredadas, dada la importancia de la Casa de Aranda, sus posesiones y los ilustres linajes emparentados con ella hasta el siglo XVII. Junto a los pretendientes masculinos es necesario resaltar a dos mujeres importantes en la trayectoria sucesoria y vital de la condesa de Aranda. Se trata de dos destacadas damas de linajes emparentados, que defendieron sus derechos sucesorios a la mencionada Casa durante todo el reinado del monarca Carlos II y comienzos del de Felipe V, hasta la defunción de la propia condesa Felipa Clavero.

Nuestro trabajo tiene por tanto como estudio la complicada trayectoria de esta viuda, en un momento fundamental del condado de Aranda, donde confluyen irreconciliables intereses, en un océano de pleitos forales en el que sobresale la personalidad y obra de esta condesa en el largo litigio sucesorio del seiscientos. Este espectacular proceso, tanto por su duración en el tiempo como por el número de juicios desatados, destaca la dura y larga defensa de los derechos legítimos de esta viudez, que queda sin resolución hasta la muerte, ya en el siglo XVIII.

Este caso concreto puede resultar esclarecedor para desentrañar vertientes comunes en las situaciones de las viudas de la nobleza y las circunstancias particulares, que por sus propias características concretas y la magnitud de las querellas interpuestas, aúnan los factores jurídicos comunes a la situación de las mujeres nobiliarias, con otros de gran interés histórico como el patrimonio jurídico y foral, y de género, que concluyen, de forma rotunda e inapelable, en la pérdida de derechos legítimos.

Este proceso que estudiamos pone de manifiesto, cómo la condición de una viudez nos lleva al conocimiento de otros factores decisivos, que van más allá de la condición social y las características propias, y que nos abre criterios externos pero ineludibles en el caso estudiado. Me refiero a factores de todo tipo que inciden en el espacio femenino y en el marco nobiliario del Antiguo Régimen, tales como la vinculación política de la nobleza con la monarquía, las relaciones de las casas nobiliarias con el poder regnícola; la fuerza de las redes sociales en litigio dentro de cada territorio histórico; la específica legislación foral regnícola, para concluir con el legado jurídico de la propia Casa nobiliaria troncal. Todas ellas intervinieron en los derechos sucesorios de una mujer concreta, y globalmente de los problemas concretos del género en el espacio nobiliario del Antiguo Régimen. Todos ellos interceptaron los derechos de viudedad de Felipa Clavero y Sessé y, en consecuencia, los resultados finales de los esfuerzos de toda una vida para 
conseguir sus legítimas aspiraciones. Son cuestiones aparentemente específicas y particulares, pero que deben tenerse en cuenta para conocer la historia de género en su totalidad, factores y condicionantes familiares y regnícolas, que afectaron a muchas mujeres de la nobleza, con mayor o menor incidencia.

\section{La V condesa de Aranda}

Don Antonio Ximénez de Urrea, Alagón y Espés, V conde de Aranda y de Sástago, había fallecido el 15 de febrero de 1654 en las casas del Palacio de Épila, en su propia habitación, llamada de la Cuadra. Así lo testifico el notario Juan Antonio Pérez, que lo encontró tendido en su cama y vestido de capuchino, con la cara descubierta y carente de vida. Se hizo acto público de su muerte con presencia constatada de varios personajes ilustres ${ }^{3}$. Debía de ser enterrado en la cisterna del presbiterio de la Iglesia de Épila, con unos funerales solemnes, propios de su dignidad. Estos funerales se realizaron en todas las parroquias de sus estados, celebrándose en ellas 30.000 misas $^{4}$. Este importante noble aragonés, además del condado de Aranda y el de Sastago, era Vizconde de Biota, señor del Vizcondado de Rueda, de la Tenencia del Alcalatén y de otras villas del reino de Valencia.

A partir de este momento la vida de su viuda, doña Felipa Clavero y Sessé, se vio alterada durante sucesivos decenios por su controvertida herencia. Así fue relatada por ella misma y dejó constancia repetidas veces en la abundante correspondencia enviada a diferentes personajes de la vida social y política, incluido el propio monarca Carlos II Habsburgo, a lo largo de toda su vida, años que abarcaron los del propio monarca, y quien fue partícipe de la situación de la Casa de Aranda desde su acceso al trono hasta el fin de sus días. La condesa Felipa aún sobrevivió unos años al rey, y muestra de ello fue su activa dirección en la participación de las obras militares en Épila durante la Guerra de Sucesión en 1705.

En las cartas mencionadas relataba el largo y proceloso mar de sus pleitos en diversos reinos, tribunales e instancias, sostenidos primero por la sucesión del condado en favor de un Ximénez de Urrea y Fernández de Heredia, además de otros descendientes dispuestos a disputar por la herencia de la Casa de Aranda, como explicaremos brevemente después, y luego, en el seno de la familia por la marquesa de la Viñuela ${ }^{5}$. Una vida calificada por Felipa Clavero Sessé como «de continuadas lágrimas». Todo ello, motivado según la V condesa por: «tanto por la ambiciosa aplicación de las partes, quanto por el sumo aprecio de honor, y jurisdicciones, que en si contienen hasta el último carácter de una grandeza» ${ }^{6}$.

3 Archivo Histórico Provincial de Zaragoza [en adelante AHPZ], S. IV, leg. 113, 28-1.

4 AHPZ, S. IV, leg. 113, 28-1.

5 Archivo Histórico Nacional [en adelante $A H N$ ], leg. 6.804, nº 385.

6 Manuscrito original: Dona Felipa Clavero y Sesse, Condesa de Aranda, Viuda del Conde Don Antonio Ximenez de Urrea, por cuya muerte sin sucession se originó los graves pleytos que sobre la de sus Estados, y bienes libres han fatigado los Tribunales de los Reynos de Aragon, y Valencia. $4^{\circ}$. 6 num. Bll. Br. mit Rückenfalz. Colección privada: a través de Antiquariat MEINDL E SULZMANN OG. 


\section{Felipa Clavero y Sessé: la mujer}

Felipa Clavero Sessé era aragonesa, nacida en Zaragoza en 1620, vivió allí durante gran parte de su vida, excepto los años que compartió con el $\mathrm{V}$ conde de Aranda, transcurridos en la casa Palacio de Épila. En esta mansión pasó largas temporadas con su hermana Serafina Ángela Clavero a la que el conde de Aranda, su cuñado, regaló en su testamento 1.000 escudos de oro para una valiosa joya. Ambas hermanas compartieron juntas una importante etapa de su vida, siendo Serafina la mayor, con una diferencia de escasos cinco años ${ }^{7}$.

El padre de la condesa, Francisco Clavero, aunque nacido en Madrid, fue Caballero hijosdalgo del reino de Aragón (Castán, 2016). Los Clavero constituyeron una familia infanzona, afincada en los primeros ascendientes en la localidad oscense de Fornillos desde el siglo XVI. Con el tiempo se extendieron y aparecen en las villas de Lascellas y Royuela. Francisco Clavero se casó con María Francisca Sessé y Sessé, también de familia infanzón originaria de los valles de Abena y Solana, apellido constatado en una estirpe que se remonta al siglo XI, y que luego se extendió por importantes poblaciones aragonesas. El padre de la futura condesa de Aranda ejerció el cargo de gobernador del Castillo de Essen.

Felipa Clavero y Sessé alcanzó la viudez dos veces a lo largo de su longeva vida. Casó en primeras nupcias con Antonio Xímenez de Urrea, conde de Berbedel, Caballero de Aragón. Fue en el segundo matrimonio cuando se desposó con el V conde de Aranda y de Sástago, Antonio Ximénez de Urrea. En este segundo enlace con el último titular de la casa de Aranda no tuvo hijos. Sin embargo, en sus primeras nupcias con el conde de Berbedel, dio lugar a una ilustre descendencia. Sus hijas, María Apolonia y María Antonia emparentaron con dos reconocidos linajes, surgidos de ventajosos matrimonios acordados. Ambas dotaron a sus descendientes con importantes posesiones territoriales, algunas radicadas en tierras de Valencia.

María Apolonia Ximénez de Urrea casó con el valenciano Jorge de Castelví, también conde de Carlet. Un exponente reconocido de la nobleza titulada valenciana. Su hijo Felipe de Castelví y Urrea, fue quien heredó el condado de Carlet, y extendió la grandeza familiar con su unión con Ana de Escriba e Hijar, de la que tuvo dos hijas, Josefa y Ana de Castelví. La otra hija de Felipa Clavero, María Antonia Ximénez de Urrea, casó con Melchor Portocarrero, conde de Monclova, cuyo descendiente, Antonio Portocarrero y Urrea, heredó el condado de Castelví, también de notable prosapia valenciana. Su descendencia fueron cuatro hijos: Inés, Josefa, José y Baltasar Portocarrero.

No debe pasarse por alto la importante descendencia de Felipa Clavero Sessé en tierras valencianas, así como las posesiones que el V conde de Aranda legó en sus cláusulas testamentarias, así como los posibles intereses que esta rama del condado de Berbedel mantendrá en las posesiones territoriales de los Aranda en el reino de Valencia. Estas nuevas familias también contemplaron con interés la herencia de la V condesa de Aranda. Posiblemente este emparentamiento de las dos hijas del 
primer matrimonio de Felipa Clavero y Sessé con importantes linajes valencianos, suscitaron los recelos de los Fernández de Heredia y su expansión por la tenencia del Alcalatén, entre otros bienes valencianos, que terminaron por amargar los últimos años de vida de la condesa de Aranda.

Felipa Clavero y Sessé dejó este mundo transcurrido ya un decenio del siglo de las luces. Otorgó testamento el 8 de noviembre de 1710, falleciendo a las 10 de la mañana del 1710. Sus últimas voluntades fueron leídas en Madrid el 7 de noviembre. Fue enterrada en la Iglesia de las religiosas de su convento de la Purísima Concepción de Nuestra Señora de Épila, junto a su segundo marido, el V conde de Aranda ${ }^{8}$.

\section{Los condicionantes forales de los litigios judiciales en Aragón}

La lucha judicial de la condesa de Aranda comenzó nada más fallecer su marido en 1654. Unos pleitos de ingente magnitud documental cubrieron los años siguientes, que dieron como resultado el traspaso de sus principales propiedades a favor de Pedro Pablo Ximénez de Urrea, Zapata, Fernández de Heredia, en la calidad del título de VI conde de Aranda.

El caso concreto de las dudas sobre la herencia del V conde de Aranda tienen su origen en dos factores específicos de la evolución jurídica familiar.

La primera se articula en las condiciones impuestas a la calidad otorgada a la viudez de la condesa, hechas en las capitulaciones matrimoniales en los momentos de la preparación notarial del matrimonio. La segunda se centra en la propia constitución del linaje de los Ximénez de Urrea, y en los cambios ocasionados en la transmisión de las propiedades y, sobre todo, especialmente en la constitución del mayorazgo de los Ximénez de Urrea, y además de los problemas derivados en la sucesión del mismo. Todos ellos son fundamentales para situar la condición de esta viuda, no sólo para el futuro de la Casa de Aranda, sino para el estudio que nos ocupa: el conocimiento de los derechos de las viudas del Antiguo Régimen y los pormenores internos, esenciales para concretar las dificultades de estas mujeres y de los estudio de género.

Las condiciones sobre la futura viudedad de la condesa Felipa Clavero y Sessé se acordaron en las capitulaciones matrimoniales, firmadas en Épila el 17 de julio de $1653^{9}$. Se estableció que, si esta dama sobrevivía al conde don Antonio y no hubiese hijos fruto de esta unión, se optaría por un denominado «pacto especial», con la condición de la denominada "viudedad limitada». Este término figura así textualmente en los documentos consultados. Esta viudedad reducía sustancialmente las propiedades que podían corresponder a una legítima condesa de Aranda y Sástago y a los espléndidos Estados de los que había disfrutado hasta la fecha. De cumplirse esta fórmula de viudedad Felipa Clavero y Sessé heredaría únicamente una testimonial parte de la antigua Casa y de sus tierras, que consistiría en: la posesión de la villa de Rueda de Jalón, sus términos, rentas y jurisdicciones, y 
el Palacio de la villa de Épila, con su jurisdicción civil y criminal y sus términos, domicilio dónde habían convivido los condes durante su vida común y constituía la casa nuclear del condado.

Las capitulaciones especificaban además una condición añadida. Ésta incluía «la reserva de poder aumentar la viudedad en todos los demás bienes de sus estados, ya fuese en cualquier escritura o testamento que hiciesse, y otorgasse para dicho aumento». Esto se pactó, «que fuese parte y porción de la Capitulación matrimonial ${ }^{10}$. Este texto estuvo vigente durante toda la existencia del V conde, y no fue modificado hasta un año antes de su muerte, concretamente en 1653. Durante todos estos años la vida matrimonial de los condes no dejó descendencia.

Entre tanto el linaje de los Ximénez de Urrea, sostuvo diversos pleitos internos por las condiciones en la sucesión del patrimonio. Hasta esa fecha el vínculo de agnación masculina, fue explicitado en todas las capitulaciones matrimoniales del linaje desde Pedro Ximénez de Urrea, hechas en Épila el 8 de agosto de 1421. Como es notorio, este señorío daría lugar después a un mayorazgo regular, constituido por Lope Xíménez de Urrea en 1490 (Gómez, 1664). Volveremos después a este mayorazgo con sus formas de agnación masculina, ya que incidirá también en la viudez de la condesa Felipa.

Sin embargo en 1653 el conde Antonio Ximénez de Urrea y Sástago, por esta facultad implícita en sus capitulaciones matrimoniales «modificó la herencia otorgada previamente a su mujer y le aumentó la viudedad a la posesión de todos los Estados de la Casa de Aranda» $\mathrm{y}$ «en los demás bienes le nombró heredera» (Díez de Aux, 1664). A esta viudedad se le denominó «viudedad completa» $\mathrm{O}$ «viudedad universal» (Díez de Aux, 1664: 92, 93). Según esta adicción del testamento su viuda, Felipa Clavero y Sessé, heredaba la parte substancial de la Casa de Aranda, y en las demás propiedades bienes y rentas se quedaba en calidad de heredera, salvo por las prescripciones jurídicas particulares, que los propios bienes arrastraran consigo, fruto del constitucionalismo foral aragonés implícito en cada posesión inmemorial, que arrastraba unos vínculos nobiliarios específicos.

En este aspecto merece la pena dedicar unos breves párrafos a la evolución de la constitución patrimonial de los Ximénez de Urrea. Miguel Ximénez de Urrea, en sus capitulaciones matrimoniales, dadas en Épila en 1493 para su matrimonio con Aldonza de Cardona, continuó con la sucesión de la agnación de los hijos varones, pero abrió la compuerta al especificar que, a falta de ellos, podrían heredar el vínculo las hijas del matrimonio y sus descendientes. Este cambio en la condición hereditaria de los bienes, no llegó a materializarse, pero constituyó un precedente en el linaje. Su hijo Fernando Ximénez de Urrea heredó el legado familiar y en sus pactos nupciales con Juana de Toledo, dados en Zaragoza el 14 de febrero de 1529, obtuvo la legación de su padre y retorno al vínculo para sus hijos y descendientes por línea recta masculina.

Esta nueva condición de traspasar el título y el mayorazgo a los hijos varones, y si no los hubiese a los otros familiares por estricta línea sucesoria masculina, fa- 
voreció en el momento al quinto descendiente dentro del entorno del parentesco. Y este fue Pedro Pablo Ximénez de Urrea, Zapata, Fernández de Heredia. El salto del linaje era evidente de los Ximénez de Urrea se daba paso a los Fernández de Heredia, familia aragonesa que había experimentado un espectacular ascenso social en las dos generaciones, anteriores al evento fúnebre (Ximénez de Urrea, 1661).

Este noble aragonés inicio el proceso judicial titulado Alegación en fuero y derecho por el Ilustrissimo señor don Pedro Pablo Ximénez de Urrea Zapata, Fernández de Heredia, Governador y Capitán General del Reyno de Aragón y el Ilustrissimo señor marqués de Cañizar (Ximénez de Urrea, 1661). El enfrentamiento entre los posibles pretendientes a la herencia del V conde de Aranda llevó primeramente a una disputa entre partes en un pleito civil ordinario, cuestionándose en el momento la presentación formal del testamento, las circunstancias de su ejecución y el valor de su firma, en un interminable debate sobre la legislación foral aragonesa contenida en el ordenamiento jurídico del reino. La sentencia pronunciada por la institución colegiada del Justicia de Aragón ratificó la validez del testamento el 20 de diciembre de 1663, nueve años después del óbito del conde.

Esta primera sentencia fue denunciada también por Juana de Toledo, bisabuela del conde, en calidad de madre y tutora de su hijo, el conde Juan, y los derechos de la familia Ximénez de Urrea a la descendencia troncal. Su argumentación volvió a retomar el conjunto de los derechos familiares pero sobre todo la validez de las últimas voluntades de su marido, el conde Fernando, quien obtuvo la aprobación de su testamento tras tres pleitos y tres sentencias a su favor, en los tres tribunales de Justicia mayores del Reino, Audiencia Real y Corte del Justiciazgo (Ximénez de Urrea, 1661). Su pretensión fue estimada en aquel momento a favor del testamento del conde Fernando, y por tanto de la familia Ximénez de Urrea. El desenlace de tan transcendental decisión en los principales tribunales regnícolas y sobre la herencia de la misma Casa y Estado de Aranda, favoreció aparentemente la validez del testamento del conde Antonio Ximénez de Urrea. Esta sentencia, que favorecía con mejores circunstancias los derechos de viudez de su mujer, Felipa Clavero y Sessé, funcionó por poco tiempo (Ximénez de Urrea, 1661).

Este primer pleito civil ordinario dio como resultado la resolución favorable a los derechos de viudedad de Felipa Clavero y Sessé por la Institución del Justiciazgo aragonés. Fue pronunciado válido el testamento y «verdadero y formal ya que contenía la solemnidad foral» (Ximénez de Urrea, 1661). Ese mismo día en que se dio la sentencia, el 20 de diciembre de 1663, apeló contra ella el pariente más próximo a la línea de sucesión del mayorazgo, que se había manifestado contrario al testamento del conde Antonio ya el 2 de septiembre de 1654: Pedro Pablo Ximénez de Urrea, Zapata, Fernández de Heredia. El gobernador general por el derecho notorio a la Casa de Aranda, y el marqués de Cañizar por ser parte formal de la Casa de Sástago, pretendiéndola por los vínculos del mayorazgo correspondiente, como hemos indicado anteriormente

La denuncia constituyó una alegación contundente sobre la formulación del testamento, basándose únicamente en aspectos circunstanciales y de forma, como los actos de entrega, el modo del apercibimiento y la denominada carta pública de 
muerte. Todo ello fue justificado y fundamentado en la recopilación de los fueros aragoneses y en concreto del denominado Proceso Iannis de Almelda, Super Compulsa (Ximénez de Urrea, 1661).

En este primer proceso fue firmante también otro de los pretendientes Carlos Alagón y Espés ${ }^{11}$, (Ximénez de Urrea, 1661). Como es lógico suponer la alegación incluía al futuro sucesor del condado de Sástago, que debía continuar también con su sucesión agnada.

A pesar de los diferentes candidatos y de la duración del pleito civil ordinario durante nueve años, la sorpresa judicial llegó de la mano de uno de los Lugartenientes de la Corte del Justiciazgo aragonés, quien había valorado y firmado previamente la validez del testamento del conde Antonio en la primera sentencia y en consecuencia las cláusulas de la viudez de la condesa Felipa Clavero y Sessé. Este lugarteniente de la Corte del Justiciazgo declaró finalmente el testamento como «falso e insolemne», mediante la argucia jurídica de un Decreto de Firma, que ejecutó solo y sin contar con el apoyo ni aprobación del Consejo de la Lugartenencia, que no intervinieron formalmente ni llegaron a ver la petición. El lugarteniente autor de este decreto fue Miguel Mateo Díez de Aux, relacionado familiarmente con Pedro Pablo Ximénez de Urrea (Corona, 2015: 14-15) ${ }^{12}$.

Por el otro lado, tras la demanda de la condesa Felipa actuó Tomás Casimiro Clavero y Sessé, caballero del reino, quien solicitó un Apellido de Aprehensión, pliego de revisión, que después de 9 años fue revocado. Este nuevo paso legislativo desacreditó el valor de la Sentencia judicial previa a favor de los derechos de viudedad contenidos en el testamento, pero en cambió ratificó los de los familiares incluidos en el tronco de la sucesión del mayorazgo de los Ximénez de Urrea.

Inmediatamente salió a colación el grado de parentesco que unía al lugarteniente autor del Decreto de Firma, con el beneficiario final de esta primera parte de la sucesión al condado de Aranda: Pedro Pablo Ximénez de Urrea Zapata Fernández de Heredia. Los comentarios legales de las dos partes llenan un dilatado expediente, dónde se demuestra la injusticia cometida con la invalidación del testamento del V conde de Aranda y con ello, el reconocimiento de la viudez de la condesa de Aranda, Felipa Clavero Sessé. Alegatos de esta índole llenan los folios de la recusación, recogemos algunos de ellos que prueban la injusticia que se cometió, a pesar de los numerables comentarios de los juristas del Justiciazgo:

Cuando de acuerdo se comete un contrafuero y se hace agravio con injusticia notoria, no queda en términos de culpa de olvido (que aquí no lo pudo ver) passa y trasciende a términos de dolo porque así lo presume el derecho (Díez de Aux, 1664: 46).

Además, el informe añadía los daños que esta sentencia causaba colateralmente a la condesa Felipa:

11 Olim. Carlos de Espés, olim. Urrea y Alagón.

12 Aunque era pariente, estaba fuera del cuarto grado, parece que se encontraba en muy primer grado de parentesco. 
No sólo hace influencia el daño que resulta a la Sra condesa de Aranda por los efectos que obra en Aragón, más aún por los que ha obrado en el Reino de Valencia donde litiga, aunque responde al Sr. Lugarteniente que las firmas no deben ser obedecidas por los tribunales de Valencia porque no se dilata su jurisdicción fuera de los límites de este Reino (Díez de Aux, 1664: 48).

Para los representantes del mundo jurídico aragonés este Proceso supuso un repaso legislativo de la mayoría de los foralistas y tratadistas consumados del derecho regnícola, y así lo esbozaron a lo largo de los 180 folios trabajados, resaltando para el futuro el debate habido entre los denominados Procceso civil ordinario, Procceso Dr. Thomás Clavero, Processo Ioannis de Almelda y Super Compulsa, Texto de Inhibición de firma, del 22 de diciembre de 1663, con la Revisión y Firma, del 3 de enero de 1664 (escrito todo por el doctor Joseph Panzano: 180 folios y 292 alegaciones). Tras el proceso civil ordinario la condesa Felipa Clavero y Sessé recurrió a la Real Audiencia del reino de Aragón, esperando ser ratificada como viuda heredera. Recurrió en otro proceloso pleito.

Entre medio los derechos de viudedad de la condesa surgían en la práctica señorial aragonesa. El convento de las monjas de la Purísima Concepción de la villa de Épila con su abadesa y las monjas capuchinas reclamaban ciertas cantidades de censales cargados e impuestos a las comunidades de Calatayud, Daroca y Teruel, que se inventariaron en poder de la condesa en virtud de una de las cláusulas del testamento del conde don Antonio. Quedaban cantidades por pagar de época del $\mathrm{V}$ conde, y la condesa como heredera y teórica beneficiaria del testamento debía proceder al cobro y con ella las monjas. Una vez más no fue considerada la condición de la viudez y el beneficiario volvió a ser Pedro Pablo Ximénez de Urrea, Zapata y Fernández de Heredia. Éste reclamó el pago de estos censales, en calidad de pariente más cercano por la línea paterna del V conde de Aranda, beneficio fori, y la otra mitad la solicitó (Fernández de Heredia, 1664).

\section{El problema de toda una vida: los derechos de viudedad de Felipa Clavero y Sessé}

En 1694 Felipa Clavero y Sesé, condesa de Aranda, recordaba a Carlos II Habsburgo el asunto que había remitido a la real consideración en varias ocasiones a lo largo de su vida. Se trataba de un tema transcendental para ella, como era su viudez y la sucesión legítima a los Estados de la Casa de Aranda, tanto en Aragón como en tierras valencianas. Ella se quejaba de la actitud del monarca al conocer los sucesivos memoriales que le había ido enviando a lo largo de los años, del que solo había merecido el «consuelo de la mayor dilación en la resolución de los mismos». En su opinión tanta tardanza había ocasionado una mayor lentitud en la tramitación de los pleitos, con el resultado del aumento del «careo» entre ella y la marquesa de la Vilueña. El rey le había correspondido durante años con un infeliz silencio ${ }^{13}$.

En esta ocasión recordaba cómo habían transcurrido 40 años desde que murió su marido don Antonio, el último descendiente legitimo del estado de Aranda, con

13 Manuscrito original: Dona Felipa Clavero y Sesse, Condesa de Aranda... véase nota 5.

ASPARKÍA, 30; 2017, 17-28 - ISSN: 1132-8231 - DOI: HTTP://DX.DOI.ORG/10.6035/ASPARKIA.2017·3O.1 
los perjuicios habidos desde entonces por el retraso en la concesión de su derecho de viudedad sobre todas las posesiones y tierras primero en el reino de Aragón y luego en el de Valencia. Calificaba su situación como una «desconsolada viudez», en la que no le dejaban tregua, ni el menor respiro, los insidiosos juicios que, en los tribunales de estos dos reinos, tenían interpuestos con diversas causas los diferentes pretendientes a los estados de Aranda, y más concretamente las otras dos mujeres emparentadas con miembros vinculados en diferentes grados con la Casa de Aranda. Una dama era la viuda del sucesor de su marido en el condado, el VI conde, y últimamente, los de la otra viuda, Juana Rocafull y Rocabertí, esposa del VII titular, conocida como la marquesa de la Vilueña.

Esta dama era la viuda del marqués Dionisio Ximénez de Urrea, autonominado como VII conde de Aranda. Este noble aragonés ostentaba el título del marquesado de la Vilueña. Al fallecer antes que su esposa, Juana Rocafull y Rocabertí, esta permaneció viuda mucho tiempo después del óbito de su marido, y había testado sus posesiones a favor de su único hijo ${ }^{14}$.

El marqués de la Vilueña era el varón descendiente legitimo del reconocido como VI conde de Aranda, Pedro Pablo Ximénez de Urrea. Era el fruto de su matrimonio con $\mathrm{M}^{\mathrm{a}}$ Josefa de Vera, quien incorporo las propiedades y títulos, de la descendencia femenina, aunando también los de los Fernández de Hijar y Camargo, agrupando todos en sí un legado sanguíneo de acrisoladas casas aragonesas.

En sus últimas voluntades, Pedro Pablo Fernández de Heredia, fechadas el 27 de mayo de 1689 en Zaragoza, dejaba como heredera a su mujer, María Josefa de Vera y Claver, Fernández de Híjar y Camargo, y ésta lo manifestaba en favor de su hijo don Dionisio Ximenez de Urrea Zapata y Fernández de Heredia ${ }^{15}$.

Todos estos acontecimientos, con los consecuentes procesos de sucesión y pleitos, abarcaron la vida de Felipa Clavero Sessé, desde que accedió a su estado de viudez, en los que fue calificada como «La vieja condesa de Aranda».

En resumidas cuentas, tres viudas, una del último titular del condado de Aranda y Sástago y la otra, del VI conde de Aranda del linaje de los Fernández Heredia, y la otra viuda del único descendiente directo del VII conde de Aranda, se disputaron los estados de la Casa en Aragón y Valencia. Tres reputadas mujeres estaban enfrentadas judicialmente por los derechos sobre uno de los estados de mayor solera de la Corona de Aragón.

\section{Conclusiones}

La biografía vital de la condesa de Aranda perfila rasgos de una innegable personalidad como mujer, a la que se unen saberes femeninos nobiliarios notables, y un conocimiento de la realidad institucional y jurídica verdaderamente sobresalientes. Conjuga a la vez una sabiduría precisa del funcionamiento del aparato político regnícola fuera de lo común, tanto del reino de Valencia como de Aragón. 
A todo esto, ya de por sí notable, hay que añadir sus precisos conocimientos del aparato administrativo del régimen monárquico de los Austrias, manifestados en sus memoriales y cartas al rey Carlos II, cuya lectura y estudio aplazo para otro momento.

Todo ello queda manifiesto en su abundante correspondencia con juristas y asesores. La correspondencia, tanto pública como privada, es abordada a través de su asesor y secretario, a la que añade sus puntos de vista y criterios, que realiza de su puño y letra, aspecto que ratifica, sin lugar a dudas, su posición personal. En ellos se manifiesta la claridad de su mente y la calidad de sus criterios y pensamientos, que quedan plenamente expuestos con la soltura y decisión manifestada en los sucesivos y complicados procesos judiciales y vitales.

Estas consideraciones observadas en la V condesa de Aranda, Felipa Clavero y Sessé, abren las puertas para estudiar más a fondo la educación y preparación intelectual de las mujeres de la nobleza, con sus habilidades políticas y epistemológicas, a las que añadimos saberes de alcurnia inculcados desde la niñez para desarrollar los roles sociales requeridos en las necesidades de las élites sociales.

Por último, esta escalofriante vida dedicada a los procesos judiciales contra los posibles aspirantes a su viudedad completa (de los cuales sólo hemos relatado la mitad), nos hace reflexionar sobre valoraciones, a veces excesivamente alegres o contundentes, sobre los firmes derechos de las viudas, en contraposición a las otras condiciones sociales de las mujeres. El Antiguo Régimen a veces nos alerta, y nos hace reflexionar con mayor detalle en la metodología combinada de lo individual y lo colectivo, y en la invisibilización y la visibilidad.

\section{REFERENCIAS Y BIBLIOGRAFÍA}

Casaus Ballester, María José (2009): El condado de Aranda y la nobleza española del Antiguo Régimen. Zaragoza: Institución Fernando el Católico.

Castán Alegre, Miguel Ángel (2016): Insaculados en las Bolsas de los Oficios de la Diputación del reino de Aragón en el siglo XVII. Zaragoza: Institución Fernando el Católico.

Corona MARzol, Carmen (2015): «El progreso social de los Fernández de Heredia hasta alcanzar el Condado de Aranda: un modelo programático de ascenso nobiliario aragonés (siglos XIV-XVII)», Millars. Espai i historia, $\mathrm{n}^{\circ} 38$, Castellón de la Plana, Universitat Jaume I, pp. 13-35.

Díez De Aux, Miguel Mateo et al. (1664): Publica alegacion en fuero y derecho por la excelentíssima Señora D. Felipa Clavero y Sesse, condesa de Aranda en la denunciacion contra.... Zaragoza: Juan de Ybar.

Fernández de Heredia y Ximénez de Urrea, Pedro Pablo (ca. 1664): Por el señor Conde de Aranda, Don Pedro Ximénez de Vrrea Fernandez de Heredia y Zapata, del Consejo de Guerra de su Magestad. Con la señora Condesa viuda, doña Felipa Clauero y Sesè. En el proceso, y causa de la Abadesa, monjas, y Conuento de la villa de Epila... 
Gómez Raxo, Juan Baptista et al. (1664): Información uirídica, y foral por los señores doctores... contra la denunciación que se les ha dado por el Excelentísimo Señor Don Pedro Pablo Ximénez de Urrea, Zapata, Fernández de Heredia, Conde de Aranda. Zaragoza: Herederos de Pedro Lanaja y Lamarca.

KagAN, Richard L. (1991): Pleitos y pleiteantes en Castilla, 1500-1700. Valladolid: Junta de Castilla y León, Conserjería de Cultura y Turismo.

NAvArro Espinach, Germán (2009): «La formación de los señoríos del condado de Aranda». En María José Casaus Ballester: El condado de Aranda y la nobleza española en el Antiguo Régimen. Zaragoza: Institución Fernando el Católico, pp. 65-84.

Piedrafita y Albis, Juan Antonio (1664): Invectiva publica, Iuridica, y Foral. En defensa del ilustre Señor Don Miguel Mateo, Diez de Aux. Zaragoza: Diego Dormer.

UtRILla Utrilla, Juan F. (1999): «De la aristocracia a la nobleza: hacia la formación de los linajes nobiliarios aragoneses, (1076-1276)». En La nobleza peninsular en la Edad Media. Madrid: Fundación Sánchez-Albornoz, pp. 431-478.

Ximénez de URReA, Pedro Pablo (1661): Alegación en fuero y derecho por el Ilustrissimo señor don Pedro Pablo Ximénez de Urrea Zapata... y el Ilustrissimo señor marqués de Cañizar. Madrid: Joseph Fernández Buendía.

Recibido el 22 de noviembre de 2017 Aceptado el 22 de noviembre de 2017 BIBLID [1132-8231 (2017): 17-28] 